\title{
ON THE NUMERICAL SOLUTION OF STEFAN PROBLEMS IN TEMPERATE ICE
}

\author{
By Kolumban Hutter, \\ (Fachbereich Mechanik, Technische Hochschule Darmstadt, \\ D-6100 Darmstadt, Federal Republic of Germany) \\ AMÉDÉ ZRYD, \\ (Bonnard et Gardel, Ingénieurs Conseil SA, Avenue de Cour 61, \\ CH-1077 Lausanne, Switzerland)
}

and HANS RöTHLISBERgER

(Im Länder, CH-8713 Uerikon, Switzerland)

\begin{abstract}
Freezing processes in temperate ice consisting of a mixture of pure ice with water inclusions are studied for the case that the initial amount of moisture content is uniform. By introducing a cold source at the center of the ice specimen, the cold front propagates outwards leaving behind pure cold ice with a temperature distribution dictated by the exact set-up of the cold source. The speed of the front is directly related to the water content of the temperate ice and depends essentially on the Stefan condition.

Three types of initial and boundary conditions are considered and realized in uniaxial, cylindrical, and/or spherical symmetry: (1) a metallic core at a temperature below the freezing point is initially brought into contact with the ice and the system is left free to evolve; (2) the metallic core is kept at constant temperature below freezing; (3) Case (2) is repeated with an insulating air layer between
\end{abstract} the metallic core and the ice.

\section{INTRODUCTION}

The dynamic role of the liquid-water content in temperate ice remains one of the unsolved problems in glaciology despite the fact that it has been shown to affect flow at the melting point (Lliboutry, 1976; Lliboutry and Duval, 1985) and that first theoretical models (Hutter, 1982; Fowler, 1984; Hutter and others, 1988) have pointed out its significance in the global boundary conditions. In fact, very little is known about water content in temperate glaciers. Typical values found by Vallon and others (1976) in an ice core of an alpine glacier are between 0 and $4 \%$.

Two methods have been proposed for measuring liquid water in glaciers. (i) The dielectric constant of the mixture ice-water depends on the relative presence of the two components and may serve as an indicator of water content. But this method, used for snow, is not sensitive enough and the dielectric constant may vary with other parameters such as grain boundaries or point defects. (ii) The calorimetric method consists of measuring the amount of energy needed to freeze the water in a given volume of temperate ice. It has the advantage of being very sensitive, and the results are less influenced by the other parameters. However, the use of an adiabatic calorimeter requires the extraction of an ice core. Stresses in the probe are thus changed, and this modifies the water content (Raymond, 1976).

The need for a calorimetric method for in-situ measurements (i.e. in the bulk of the glacier, without extracting a core) led us to study the problem of the propagation of a "cold wave" in an ice-water mixture. The wave front consists of a surface of phase change that separates cold ice from the ice-water mixture. Its speed depends on the imposed boundary conditions on the specimen and the water content. By determining the migration of this well-defined boundary, we may thus infer the water content in the specimen (Fig. 1).

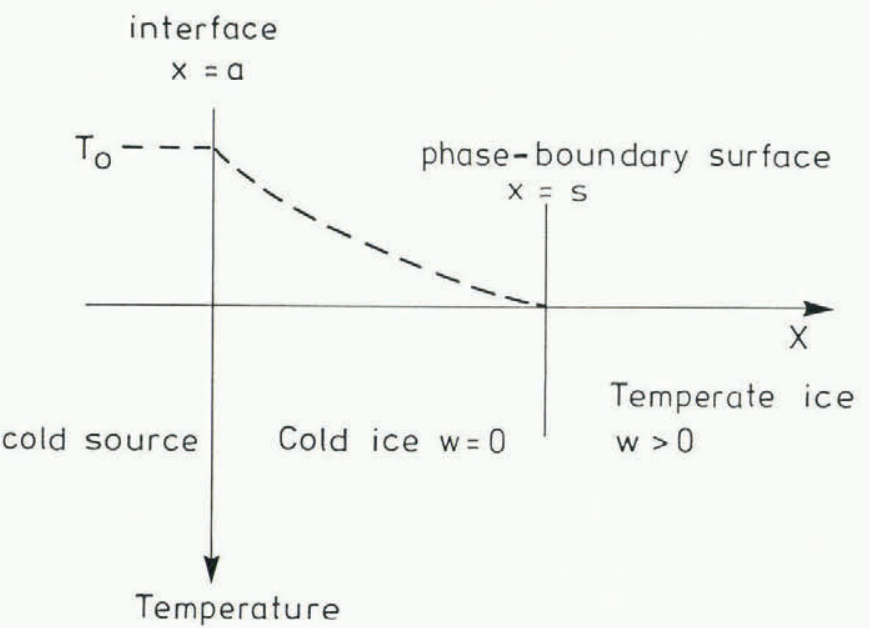

Fig. 1. Temperature of the ice and position of the interface during the experiment.

The method of determining $w$ by this procedure is an inverse problem, because its distribution must be inferred essentially from information gained along the boundary of the domain. Such problems tend to be difficult and sensitive to measurement and numerical error. Here, however, two simplifications may be justifiably invoked which make the problem tractable and, as our results show, reasonably stable to numerical error. First, length scales that are considered are a few centimetres (less than $0.25 \mathrm{~m}$ ) over which water contents in the vein system and the inclusions may be regarded as constant. Secondly, the region outside the phase-change surface may be considered to be a "bath" with uniform conditions. This bath will only affect the solution of the freezing problem at the phase-change surface. This implies that the water content should only vary very slowly over length scales of the above-mentioned $0.25 \mathrm{~m}$. Field observations lead us to be confident about these assumptions. 
Approximate analytical solutions of the solidification problem exist for one-dimensional geometries (see Vujanovic, 1989). We chose to solve the problem numerically as it seemed most suitable for the various boundary conditions we consider.

\section{THE INITIAL VALUE PROBLEM}

\subsection{Hypotheses}

Consider a specimen of temperate ice at $0^{\circ} \mathrm{C}$. Assume that a metal mass has been inserted at its center and that the contact between the metal and the temperate ice is perfect. We may consider two types of Gedankenexperiments:

(1) Assume that at time $t=0$ the metal mass is suddenly set at a prescribed temperature $T_{0}$ below the freezing point and the system is subsequently left free to evolve.

(2) Assume that at time $t=0$ the metal mass is subject to a temperature jump and that its new temperature (below freezing) is kept constant while the surrounding ice is free to adjust its thermal state to the new conditions.

In both cases, the cold will propagate from the metal into the ice and freeze the interstitial water. The cold front that forms the surface of phase change between the wholly cold ice and the ice-water mixture can be identified experimentally as the location where the temperature gradient suffers a jump and thus a kink in the temperature profile arises. Observations have shown these to be clearly identifiable.

We impose the following idealized initial conditions:

The temperate ice is an homogeneous mixture of water and ice uniformly at the melting temperature $=0^{\circ} \mathrm{C}$.

The specimen is thought of as infinitely large so as to eliminate the influence of finite boundaries.

The cold source (metal) is an infinite sheet, an infinitely long wire, or a spherical ball, i.e. linear, axial, or spherical symmetry prevails.

The metal is a perfect conductor and the contact between the ice and the metal is perfect. (This condition will be relaxed later on.)

\subsection{Equations}

Because of the spatial symmetry, only positions with $0<r<R(t)$ need be considered. The metal mass ranges from $r=0$ to $r=a$, the ice from $r=a$ to $r=\infty$, whereas the cold ice occupies the domain $a \geqslant r \geqslant R(t), R(t)$ being the position of the phase-change surface. In this latter domain, the heat-conduction equation is

$$
\rho_{\mathrm{i}} c_{\mathrm{i}} \Theta=k_{\mathrm{i}} \Delta \Theta, \quad a<r<R(t), \quad t>0
$$

where $\rho_{\mathrm{i}}$ is the ice density $\left(=918 \mathrm{~kg} \mathrm{~m}^{-3}\right), c_{\mathrm{i}}$ is the heat capacity of ice $\left(=2.12 \times 10^{3} \mathrm{~J} \mathrm{~kg}^{-1} \mathrm{~K}^{-1}\right), k_{\mathrm{i}}$ is the thermal conductivity of ice $\left(=2.2 \mathrm{~W} \mathrm{~K}^{-1} \mathrm{~m}^{-1}\right), \Theta$ is the temperature, and $\Delta$ is the Laplacian. Throughout this text, the dot represents the time derivative.

Boundary conditions have to be satisfied at the interface "cold ice-temperate ice" $r=R(t)$ and at $r=a$. At the former, the heat flow from the phase-change surface is balanced by the latent heat released by freezing, viz.

$$
k_{\mathrm{i}} \frac{\partial \Theta}{\partial n}=L_{\mathrm{i}} w \cdot \rho_{\mathrm{i}} R(t)
$$

at $r=R(t), t>0$, and

$$
\Theta=0 .
$$

Here, $L_{\mathrm{i}}$ is the latent heat of fusion, $w$ is the moisture content just ahead of the freezing front, and $\partial / \partial n$ denotes the spatial derivative perpendicular to the phase-change surface.

At $r=a$, heat flow and temperature must be continuous. However, for very small values of $a$ and a perfect conduction, we may regard the metal as a body with uniform temperature and heat capacity $V_{c} \rho_{c} c_{c}$. Here, $V_{c}$ is the volume of the metal (copper) piece. The time rate of change of the heat stored in the copper, $V_{c} \rho_{c} c_{c} \Theta$ must then be balanced by the heat flow through the contact surface with the ice,

$$
k_{\mathrm{i}} \iint_{\text {surf }} \frac{\partial \Theta}{\partial n} \mathrm{~d} S=V_{\mathrm{c}} \rho_{\mathrm{c}} c_{\mathrm{c}} \Theta,
$$

at $r=a, \quad t>0$. This condition applies to Gedankenexperiment (1). When the temperature is held constant, we have

$$
\Theta=T_{0},
$$

at $r=a, t>0$. Finally, we have the following initial conditions:

$$
\begin{gathered}
\Theta(a, t=0)=T_{0}, \\
R(t)=0)=a .
\end{gathered}
$$

To simplify the equations, we use the problem symmetries and then introduce dimensionless variables. If the cold source is a plate, a cylinder or a sphere, we will use Cartesian, cylindrical, or spherical coordinates. Equation (1) will be respectively

$$
\rho_{\mathrm{i}} c_{\mathrm{c}} \frac{\partial \Theta}{\partial t}= \begin{cases}k_{\mathrm{i}} \frac{\partial^{2} \Theta}{\partial r^{2}}, & \text { Cartesian, } \\ k_{\mathrm{i}}\left(\frac{\partial^{2} \Theta}{\partial r^{2}}+\frac{1}{r} \frac{\partial \Theta}{\partial r}\right), & \text { cylindrical, } \\ k_{\mathrm{i}}\left(\frac{\partial^{2} \Theta}{\partial r^{2}}+\frac{2}{r} \frac{\partial \Theta}{\partial r}\right), & \text { spherical. }\end{cases}
$$

Equation (2) can be written as

$$
k_{\mathrm{i}} \frac{\partial \Theta}{\partial r}=L_{\mathrm{i}} w \rho_{\mathrm{i}} \frac{\mathrm{d} R}{\mathrm{~d} t}
$$

and Equation (4) becomes

$$
\left(4^{\prime}\right)-\left(4^{\prime \prime \prime}\right) \quad \lambda k_{\mathrm{i}} \frac{\partial \Theta}{\partial r}=a \rho_{\mathrm{c}} c_{\mathrm{c}} \frac{\partial \Theta}{\partial t}
$$

where $\lambda=1,2,3$ for Cartesian, cylindrical, and spherical geometry, for which equation numbers are $\left(4^{\prime}\right),\left(4^{\prime \prime}\right)$, and $\left(4{ }^{\prime \prime \prime}\right)$, respectively. Next, we introduce the dimensionless variables $r=X_{0} x, t=t_{0} t^{\prime}, \Theta=u_{0} u, R=X_{0} s$, where

$$
X_{0}=\frac{a}{2} \frac{\rho_{\mathrm{c}} c_{\mathrm{c}}}{\rho_{\mathrm{i}} c_{\mathrm{i}}}[\mathrm{m}], \quad t_{0}=\frac{a^{2}}{4} \frac{\rho_{\mathrm{c}}^{2} c_{\mathrm{c}}^{2}}{\rho_{\mathrm{i}} c_{\mathrm{i}} k_{\mathrm{i}}}[\mathrm{s}], \quad u_{0}=\frac{L_{\mathrm{i}} w}{c_{\mathrm{i}}}[\mathrm{K}],
$$

for experiment (1) when the initial heat content is prescribed, and

$$
X_{0}=\left(\frac{k_{\mathrm{i}}}{\rho_{\mathrm{i}} c_{\mathrm{i}}}\right)^{\frac{1}{2}}[\mathrm{~m}], \quad t_{0}=1[\mathrm{~s}], \quad u_{0}=\frac{L_{\mathrm{i}} w}{c_{\mathrm{i}}}[\mathrm{K}],
$$

when, as in experiment (2), the temperature of the metal is held fixed. The problem can then be summarized by the equations given in Table I.

\subsection{Numerical solution}

We describe in this paragraph a numerical solution for the equation with cylindrical symmetry at fixed energy. The difficulty induced by the moving boundary can be avoided by use of the boundary position instead of the time as second independent variable, as shown by Garofalo (unpublished), which introduces an artificially fixed domain of integration and is possible because the position of the phase-change surface is a monotonically increasing function of time. We shall then define

$$
u^{\prime}(x, s(t))=u(x, t)
$$


TABLE I. NON-DIMENSIONAL EQUATIONS

Plate

Differential equation

$\frac{\partial u}{\partial t}=\frac{\partial^{2} u}{\partial x^{2}}$

Cylinder

$\frac{\partial u}{\partial t}=\frac{1}{x} \frac{\partial u}{\partial x}+\frac{\partial^{2} u}{\partial x^{2}}$

$\frac{\partial u}{\partial x}=\frac{\partial u}{\partial t}$

$\mathrm{BC}$

at $\quad \frac{\partial u}{\partial x}=\frac{\partial u}{\partial t}$

$x=a^{\prime}$

$\mathrm{BC}$

at $\quad \frac{\partial u}{\partial x}=\frac{\mathrm{d} s}{\mathrm{~d} t}$

$x=s$

IC

$t=0$ $\frac{\partial u}{\partial x}=\frac{\mathrm{d} s}{\mathrm{~d} t}$

$\frac{\partial u}{\partial x}=\frac{\mathrm{d} s}{\mathrm{~d} t}$ and

$$
\frac{\mathrm{d} s}{\mathrm{~d} t}=\varphi(s)
$$

where $\varphi$ is the speed of the phase-change surface. As $s(t)$ is a strictly growing function, the time corresponding to a given position is calculated by

$$
t=\int_{a^{\prime}}^{s} \frac{\mathrm{d} s^{\prime}}{\varphi\left(s^{\prime}\right)} .
$$

With the new variables $u^{\prime}, s$, and $\varphi$, the equations for the cylinder become (upon omitting the primes)

$$
\varphi \frac{\partial u}{\partial s}=\frac{1}{x} \frac{\partial u}{\partial x}+\frac{\partial^{2} u}{\partial x^{2}}
$$

for $a^{\prime}<x<s$ and $a^{\prime}<s<\infty$, subject to the boundary conditions

$$
\frac{\partial u}{\partial x}=\varphi \frac{\partial u}{\partial s}
$$

at $x=a^{\prime}$, and

$$
\begin{gathered}
\frac{\partial u}{\partial x}=\varphi, \\
u=0,
\end{gathered}
$$

along the line $x=\mathrm{s}$.

The numerical scheme for its solution is described in Appendix A.

\section{VARIABLE COEFFICIENTS AND INSULATING LAYER}

In this section, we introduce the thermal variability of the thermodynamical quantities. We also examine the effect of an insulating layer between the ice and the cold source. Only the case of a constant temperature source with cylindrical symmetry will be developed.

\subsection{Variable coefficients}

Allowing for variations of the coefficients, Equation (1) becomes

$$
(\rho c)(\Theta) \frac{\partial \Theta}{\partial t}=\frac{k(\Theta)}{r} \frac{\partial \Theta}{\partial r}+\frac{\partial}{\partial r}\left[k(\Theta) \frac{\partial \Theta}{\partial r}\right] .
$$

Equations (2), (3), and (4) remain the same as before. The dimensionless variables are identical to those of section 2.1 with the mere addition of

$$
\begin{aligned}
k(\Theta) & =k_{\mathrm{i}} k^{\prime}(u), \\
(\rho c)(\Theta) & =\left(\rho_{\mathrm{i}} c_{\mathrm{i}}\right) \cdot(\rho c)^{\prime}(u)
\end{aligned}
$$

where $\rho_{\mathrm{i}}, c_{\mathrm{i}}$, and $k_{\mathrm{i}}$ are reference values at $0^{\circ} \mathrm{C}$, and primes denote dimensionless quantities. Replacing the independent variable $t$ by the position of the moving boundary, we obtain the initial boundary-value problem (primes are again omitted)

$$
\rho c \varphi \frac{\partial u}{\partial s}=\frac{k}{x} \frac{\partial u}{\partial x}+\frac{\partial}{\partial x}\left[k \frac{\partial u}{\partial x}\right]
$$

for $a^{\prime}<x<s$,

$$
\frac{\partial u}{\partial x}=\varphi
$$


for $x=s$ and $a<s<\infty$, and

$$
u=\frac{T_{0}}{u_{0}},
$$

for $x=s=a^{\prime}$, of which a solution scheme is constructed in Appendix B.

Initial values are the same as before. In the calculation we use for $k$ and $\rho c$ the values given by Hobbs (1974, p. $360-61$ )

$k=2.1725-3.403 \times 10^{-3} \theta+9.085 \times 10^{-5} \Theta^{2}\left[\mathrm{~W} \mathrm{~m} \mathrm{~m}^{-1} \mathrm{~K}^{-1}\right]$,

$$
\rho c=\frac{k}{a}
$$

where

$$
a=8.43 \times 10^{-7}-0.101 \times 10^{-7} \Theta\left[\mathrm{m}^{2} \mathrm{~s}^{-1}\right]
$$

is the thermal diffusivity.

\subsection{Insulating layer}

We will now model the influence of an insulating air layer between the cold source and the ice. The heat transfer in the layer is supposed to be stationary. In this case, the heat flow through a layer of thickness $\varepsilon$ is, for a cylinder of unit length, given by

$$
q=-n \frac{\Theta-T_{0}}{\varepsilon},
$$

$n$ being the thermal conductivity of the layer. The boundary condition at $x=a$ now becomes (Carslaw and Jaeger, 1959, p. 19)

$$
k \frac{\partial \Theta}{\partial r}=\frac{n}{\varepsilon}\left(\Theta-T_{0}\right) .
$$

The introduction of the dimensionless variables defined by

$$
\begin{aligned}
& X_{0}=k_{\mathrm{i}} \frac{\varepsilon}{n}, \\
& u_{0}=\frac{L_{\mathrm{i}} W}{c_{\mathrm{i}}}, \\
& t_{0}=\rho_{\mathrm{i}} c_{\mathrm{i}} k_{\mathrm{i}} \frac{\varepsilon^{2}}{n^{2}}
\end{aligned}
$$

gives a system formed by Equations (13), (14) and

$$
k \frac{\partial u}{\partial x}=u-\frac{T_{0}}{u_{0}},
$$

at $x=a^{\prime}$; see Appendix B for the numerical peculiarities.

\section{NUMERICAL RESULTS}

\subsection{Test of the numerical solution}

An analytical solution for the freezing of water exists in the case of a constant temperature $T_{0}$ at $x=0$ (classical Stefen problem slightly modified for an ice-water mixture with a water content $w$ ). The cold-ice temperature $T$ is given by

$$
T=T_{0}+\frac{T_{0}}{\operatorname{erf} \xi} \operatorname{erf} \frac{x}{2(D t)^{\frac{1}{2}}}
$$

in which all variables are dimensional and where $\xi$ is given by

$$
\xi \mathrm{e}^{2} \text { erf } \xi=\frac{c T_{0}}{L w(\pi)^{\frac{1}{2}}}
$$

$D$ is the thermal diffusivity of the ice, $c$ is the heat capacity, and $L$ is the latent heat of melting. The position of the interface in time is described by the equation

$$
R(t)=2 \xi(D t)^{\frac{1}{2}} .
$$

This equation allows us to test the algorithm. The results presented in Figure 2 show that for large values of the

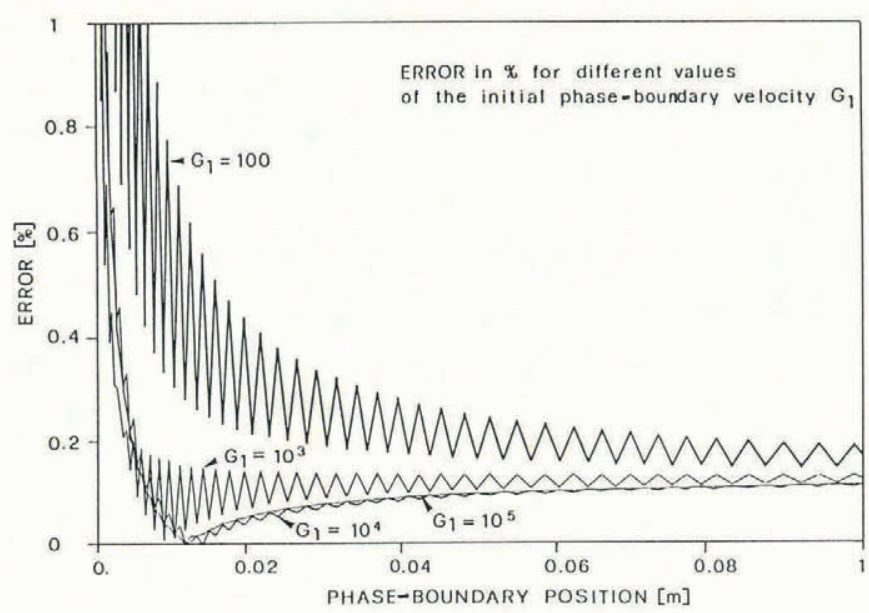

Fig. 2. Error between analytical and numerical solutions for constant coefficients and mirror symmetry: $G_{1}=$ $(d s / d t)(0)$.

dimensionless initial velocities, $G_{1}=\mathrm{d} s / \mathrm{d} t(0)$, the phaseboundary position is accurately predicted, the error being of the order of $0.1 \%$ when $x>0.01[\mathrm{~m}]$. Other tests with different values of $r, \lambda, h_{\mathrm{x}}$, and $h_{\mathrm{s}}$ (for definitions see Appendix A) have demonstrated that the most suitable values are $r=\lambda=0.5$ and $h_{\mathrm{x}}=h_{\mathrm{S}}=0.01$. However, a problem arises for very short time.

In a similar way, the equation having temperaturedependent coefficients has been tested by blocking its temperature dependence and comparing the results with runs for the constant-coefficient algorithm. The results show again a satisfactory large time behaviour with an important divergence for short time. Nevertheless, this solution can be trusted for $t>1[\mathrm{~s}]$.

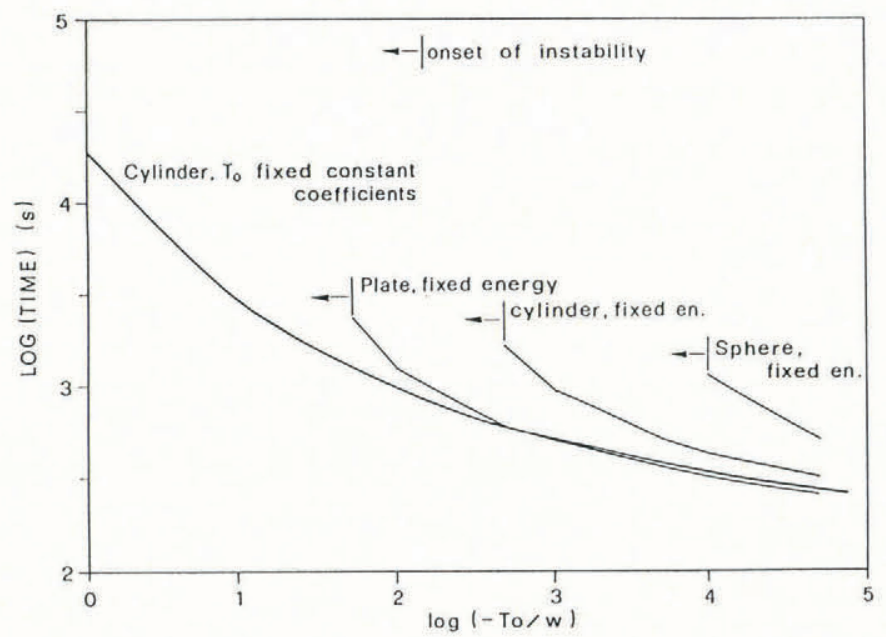

Fig. 3. Time for a displacement of the boundary of $10 \mathrm{~cm}$ as functions of $T_{0} / w$ (in $0^{\circ} \mathrm{C}$ ). Solution at constant coefficients for the cylinder with fixed temperature and fixed energy, the plate and the sphere with fixed energy. 


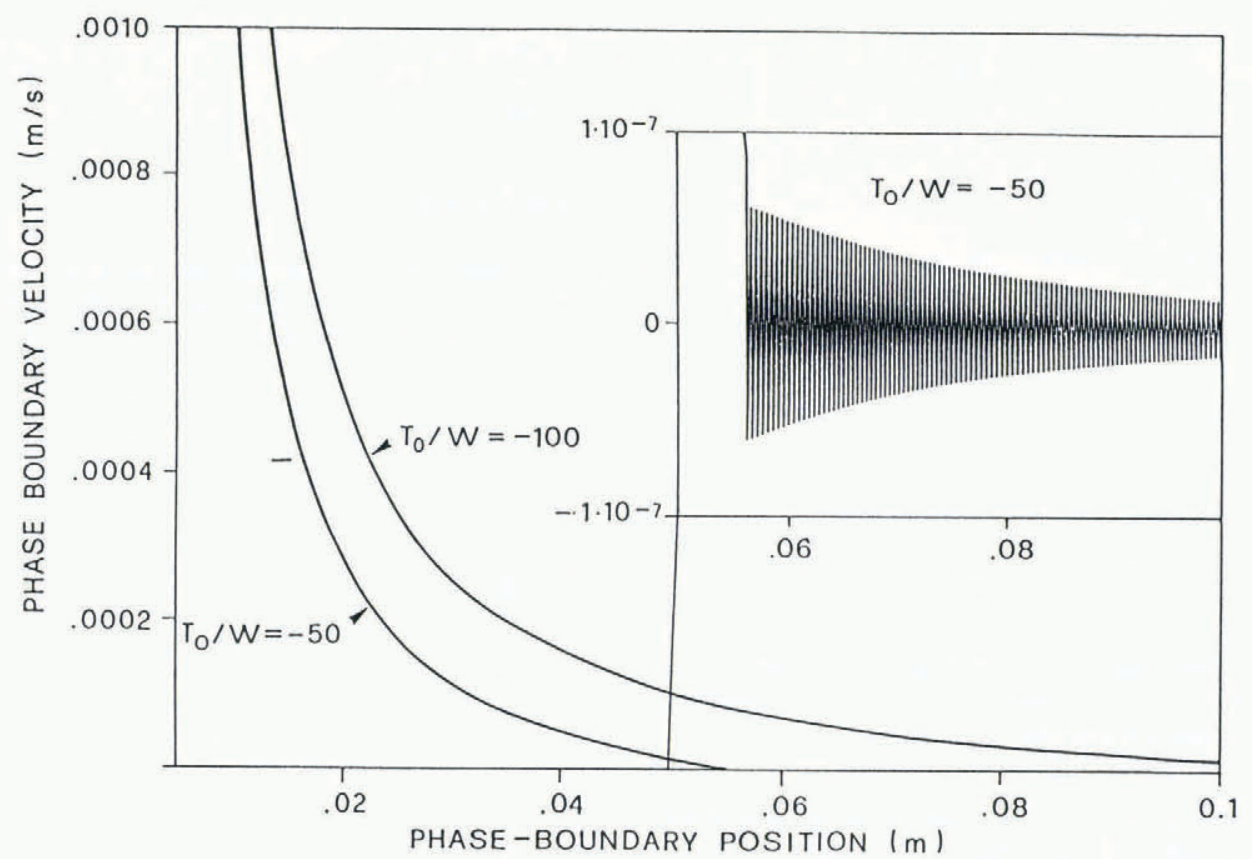

Fig. 4. Phase-boundary velocity plotted against its position. Onset of the oscillations for the experiment at fixed temperature, as shown in the inset. $T_{0} / w$ in ${ }^{\circ} C$.

\subsection{Constant coefficients}

As it appears from our scalings, we can for the case of constant coefficients combine the two parameters $T_{0}$ and $w$, and present the results as functions of $T_{0} / w$. The time required for a phase-boundary movement of $10 \mathrm{~cm}$ is given in Figure 3 for the different geometries of Gedankenexperiment (1) and for the cylindrical symmetry of Gedankenexperiment (2). Alternatively, Figure 4 shows for the experiment with a fixed initial heat content the phaseboundary velocity as a function of its position. The inset displays the continuation of the graph with $T_{0}=-50^{\circ} \mathrm{C}$ when oscillating "instabilities" arise. These oscillations are typical of Crank-Nicholson schemes and cannot be avoided, but they falsify somewhat the evaluation of the travel time of the phase boundary. The onset of such oscillations is expected whenever the available energy is very near the energy needed for the boundary displacement. It can be seen in Figure 3 that the oscillation begins at different values of the parameter $T_{0} / w$ for the plate, the cylinder, or the sphere, owing to the different amounts of energy that are needed per unit displacement of the phase boundary. The difficulty is due to the use of the phase-boundary position instead of time as an independent variable. This method breaks down when the phase boundary approaches a static equilibrium position. Under these conditions, another

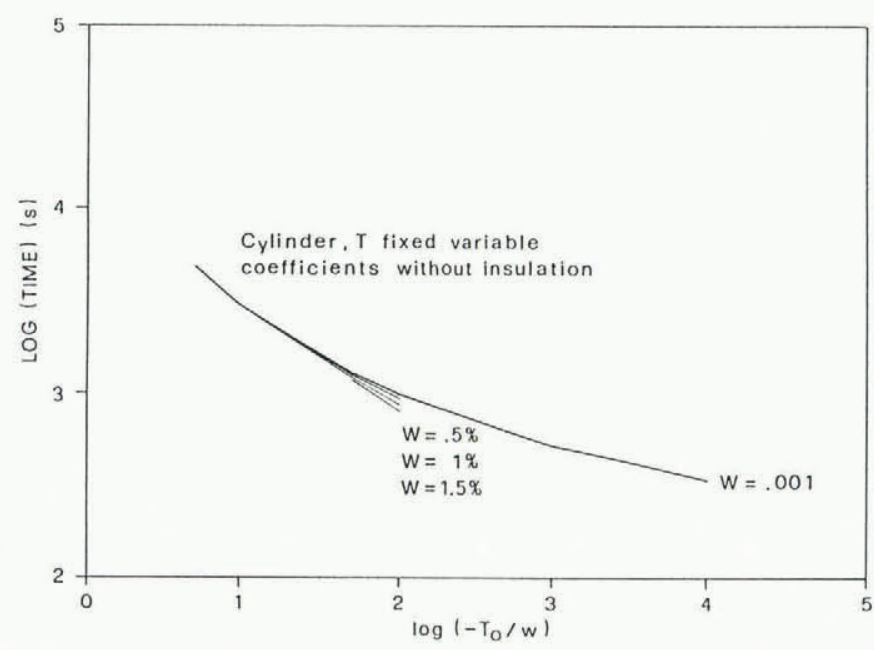

Fig. 5. Time for a displacement of $10 \mathrm{~cm}$ plotted against $T_{0} / w$ (in C) for several values of $w$. Solution for variable coefficients for the cylinder. computational technique would be more suitable (see, for example, Crank, 1984)

In the experiment at fixed boundary temperature, the computation yields convergent results at all values of $T_{0} / w$ (Fig. 4).

\subsection{Variable coefficients}

With the variable-coefficients scheme, results depend on $T_{0} / w$ and $w$; many more iterations are required for the convergence test to be successful and their numbers increase with increasing values of $\left|T_{0} / w\right|$. The maximum number of iterations is as high as 11 at the beginning of the computation, compared with 4 for the constant-coefficient equation. Moreover, the larger $w$ is, the more will the dimensionless coefficients $k$ and $\rho c$ in Equation (13) vary and thus destabilize the scheme, as has been observed by Garofalo (unpublished). But for reasonable values of $T_{0}$ and $w$, the solution is satisfactory.

For comparison with Figure 3, we display in Figures 5 and 6 the time for a boundary displacement of $10 \mathrm{~cm}$ as function of $T_{0} / w$ and for different values of $w$. Comparing Figure 5 with Figure 3, we infer that, as expected, the displacement for larger values of $w$ is more rapid if we take into account the increase of thermal conductivity with a decrease of temperature. Also of interest is the enormous

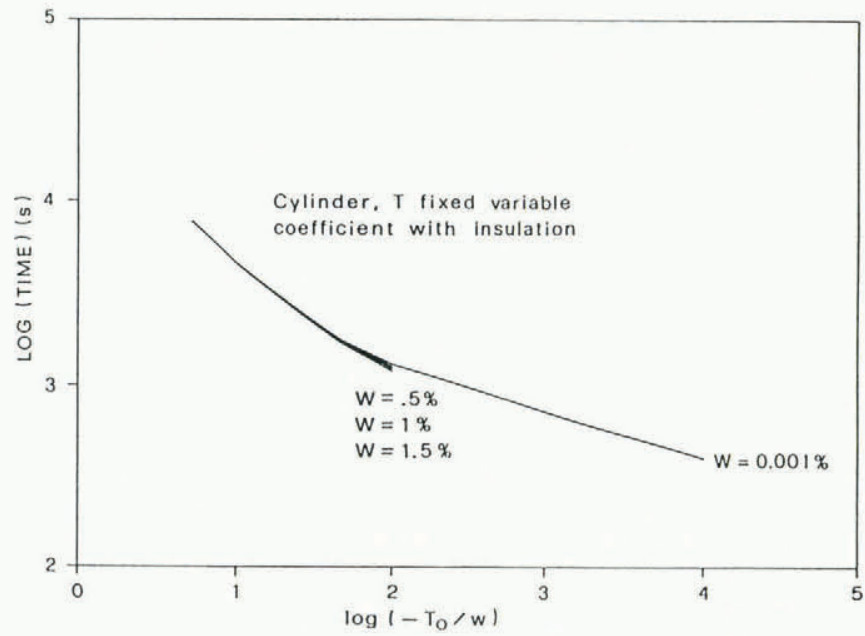

Fig. 6. As Figure 5 with an insulating layer of air of $1 \mathrm{~mm}$. 


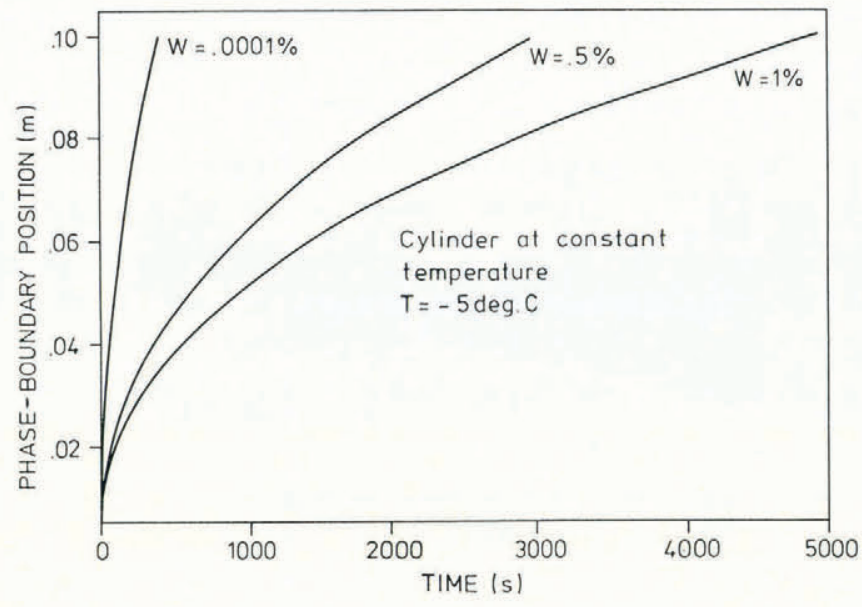

Fig. 7. Position of the boundary as a function of time for a fixed temperature of $-5^{\circ} \mathrm{C}$ and various water contents. Solution with variable coefficients.

impact of an insulating layer of air as small as $0.1[\mathrm{~mm}]$, which tends to hide the water-content effect (Fig. 7).

Figure 7 shows the phase-boundary position versus time for an initial temperature of $-5^{\circ} \mathrm{C}$ and different water contents, in the absence of an insulating layer. It can be seen that the measurement of the boundary position, as an indicator of the water content, should yield a good sensitivity, and therefore be suitable as a measuring technique.

\section{CONCLUSION}

An analytical solution for solidification has been described for various types of boundary conditions. The problem with constant coefficients gives satisfactory results. The variation of the coefficients introduces an instability at high values of the water content of temperate ice, $w$. Nevertheless, the scheme works for small values of $w$. In the view of a practical application of this problem to the measurement of the water content, we infer that this method should provide reasonable sensitivity. However, a small insulating layer between the cold source and the ice causes an important delay in the movement of the phase boundary and tends to hide the influence of the water content.

\section{REFERENCES}

Carslaw, H.S. and J.C. Jaeger. 1959. Conduction of heat in solids. Oxford, Clarendon Press.

Crank, J. 1984. Free and moving boundary problems. Oxford, Clarendon Press.

Fowler, A.C. 1984. On the transport of moisture in polythermal glaciers. Geophys. Astrophys. Fluid Dyn., 28(2), 99-140.

Garofalo, M.A. Unpublished. Numerical solution of nonlinear diffusion with a moving boundary condition. (M.Sc. thesis, University of Glasgow, 1984.)

Hobbs, P.V. 1974. Ice physics. Oxford, Clarendon Press.

Hutter, K. 1982. Dynamics of glaciers and large ice masses. Annu. Rev. Fluid Mech., 14, 87-130.

Hutter, K., H. Blatter, and M. Funk. 1988. A model computation of moisture content in polythermal glaciers. J. Geophys. Res., 93(B10), 12205-12214.

Lliboutry, L. 1976. Physical processes in temperate glaciers. J. Glaciol., 16(74), 151-158.

Lliboutry, L. and P. Duval. 1985. Various isotropic and anisotropic ices found in glaciers and polar ice caps and their corresponding rheologies. Ann. Geophys., 3(2), 207-224.

Raymond, C.F. 1976. Some thermal effects of bubbles in temperate glacier ice. J. Glaciol., 16(74), 159-171.

Vallon, M., J.-R. Petit, and B. Fabre. 1976. Study of an ice core to the bedrock in the accumulation zone of an Alpine glacier. J. Glaciol., 17(75), 13-28.
Vujanovic, B.D. 1989. A variational approach to the problem of solidification of a metal semi-infinite thermally nonlinear body. Acta Mech., 77, 231-240.

\section{APPENDIX A}

We outline a numerical solution scheme for Equations (9)-(12). Supposing that a solution $u(x, s)$ exists, we can discretize the domain as shown in Figure 8 and write

$$
\begin{gathered}
u_{i, j}=u\left(x_{i}, s_{j}\right), \\
\varphi_{j}=\varphi\left(s_{j}\right) .
\end{gathered}
$$

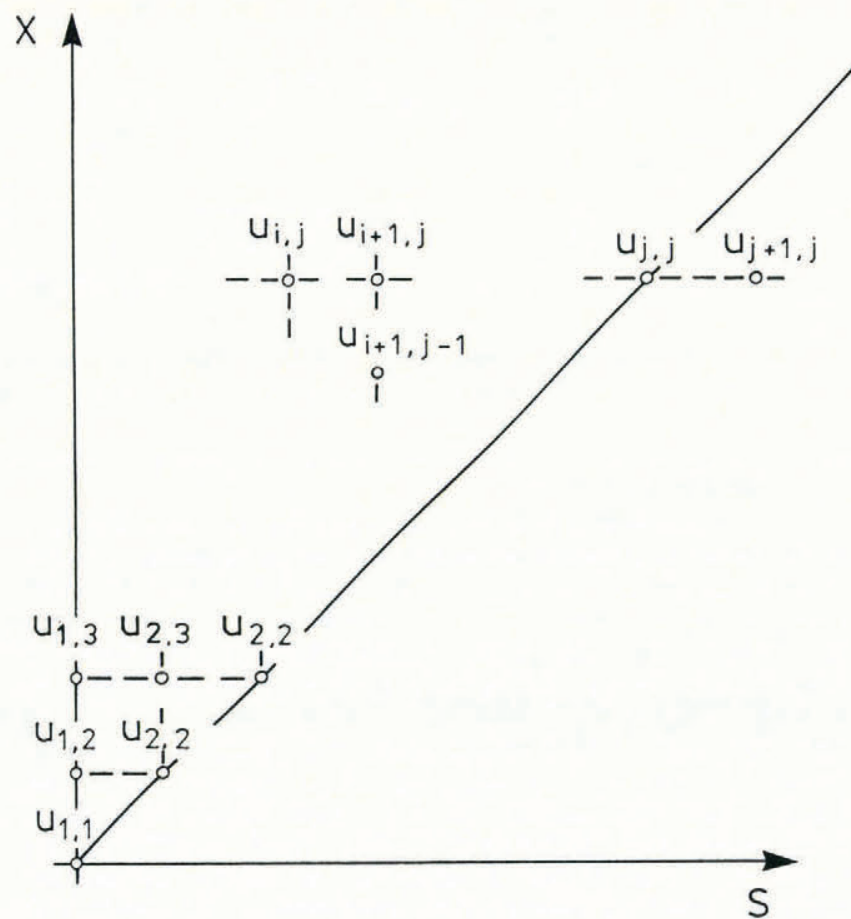

Fig. 8. Discretization of the domain.

Using finite differences, Equation (9) can now be replaced by the approximate equation

$$
\begin{aligned}
& \left(r \varphi_{j+1}+(1-r) \varphi_{j}\right) \frac{u_{i, j+1}-u_{i, j}}{h_{\mathrm{s}}}= \\
& =\lambda\left\{\frac{1}{(i+1) h_{\mathrm{x}}+a^{\prime}} \frac{u_{i+1, j+1}-u_{i, j+1}}{h_{\mathrm{x}}}+\right. \\
& \left.+\frac{u_{i+1, j+1}-2 u_{i, j+1}+u_{i-1, j+1}}{h_{\mathrm{x}}^{2}}\right\}+ \\
& +(1-\lambda)\left\{\frac{1}{(i-1) h_{\mathrm{x}}+a^{\prime}} \frac{u_{i+1, j}-u_{i, j}}{h_{\mathrm{x}}}+\right. \\
& \left.+\frac{u_{i+1, j}-2 u_{i, j}+u_{i-1, j}}{h_{\mathrm{x}}^{2}}\right\}
\end{aligned}
$$

where $r$ and $\lambda$ are two weights used to stabilize the calculation and $h_{\mathrm{x}}$ and $h_{\mathrm{s}}$ are the step sizes. This particular scheme has been used to avoid problems arising from the initial conditions. We need for this equation the value of $u_{j+1, j}$ which lies outside the computational domain. This value is approximated by using a Taylor series expansion for the function $u$, as will be explained below. After rearrangment, we obtain 
$1-\lambda u_{i-1, j+1}+\left(\Gamma_{j}+\frac{\lambda}{\Phi_{i}}+2 \lambda\right) u_{i, j+1}-$

$-\left(\frac{\lambda}{\Phi_{\mathrm{i}}}+\lambda\right) u_{i+1, j+1}=$

$=(1-\lambda) u_{i-1, j}-\left(\frac{1-\lambda}{\Phi_{\mathrm{i}}}+2(1-\lambda)-\mathrm{r}_{j}\right) u_{i, j}+$

$+\left(1-\lambda+\frac{1-\lambda}{\Phi_{i}}\right) u_{i+1, j}$

with

$$
\Gamma_{j}=\frac{h_{\mathrm{x}}^{2}}{h_{\mathrm{s}}}\left(r \varphi_{j+1}+(1-r) \varphi_{j}\right)
$$

and

$$
\Phi_{\mathrm{i}}=i-1+\frac{a^{\prime}}{h_{\mathrm{x}}} .
$$

Moreover, condition (10) at $x=a^{\prime}$ can be written as

or

$$
\frac{u_{2, j+1}-u_{1, j+1}}{h_{\mathrm{x}}}=\varphi_{j} \frac{u_{1, j+1}-u_{1, j}}{h_{\mathrm{s}}}
$$

$$
\left(\frac{h_{\mathrm{x}}}{h_{\mathrm{S}}} \varphi_{j}+1\right) u_{1, j+1}-2 u_{2, j+1}=\frac{h_{\mathrm{x}}}{h_{\mathrm{s}}} \varphi_{j} u_{1, j} .
$$

Equations (A1) and (A3) form a tridiagonal system which can be solved for the unknowns $u_{i j+1}, i=1, \ldots, j$, if the values of $u_{i, j}$ and $\varphi_{j}$ are known. The system is first solved for $\Gamma_{j}=\left(h_{\mathrm{X}}^{2} / h_{\mathrm{S}}\right) \varphi_{j} ; \varphi_{j+1}$ is then updated (we shall explain below how this is done) and the system solved again with $\Gamma_{j}$ given by Equation (A2), until the following convergence test, proposed by Garofalo (unpublished), is verified:

$$
\operatorname{ABS}\left(\varphi_{j+1}^{\mathrm{N}}-\varphi_{j+1}^{\mathrm{N}-1}\right)<h_{\mathrm{x}}^{2} \mathrm{ABS}\left(\varphi_{j+1}^{\mathrm{N}-1}\right),
$$

where $\mathbf{N}$ is the number of iterations.

To complete the solution scheme, we still need

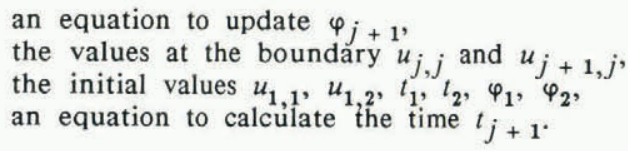

Using a Taylor series expansion for $u$ on the boundary $X=s$, we obtain

$$
u_{j, j+1}-u_{j+1, j+1}=-\left.h_{\mathrm{x}} \frac{\partial u}{\partial x}\right|_{j+1}+\left.\frac{1}{2} h_{\mathrm{x}}^{2} \frac{\partial^{2} u}{\partial x^{2}}\right|_{j+1}+\ldots .
$$

From Equations (9) and (11) we deduce

$$
\varphi \frac{\partial u}{\partial s}=\frac{1}{x} \varphi+\frac{\partial^{2} u}{\partial x^{2}} .
$$

Given that on the line $x=s$

$$
d u=\frac{\partial u}{\partial x} \mathrm{~d} x+\frac{\partial u}{\partial s} \mathrm{~d} s=0
$$

and that $\mathrm{d} x / \mathrm{d} s=1$, we infer $\partial u / \partial x=-\partial u / \partial s$ and thus obtain, in view of Equations (9) and (11)

$$
\begin{aligned}
& \frac{\partial u}{\partial s}=-\varphi, \\
& \frac{\partial^{2} u}{\partial x^{2}}=-\varphi^{2}-\frac{1}{x} \varphi
\end{aligned}
$$

on $x=s$. Thus, with condition (12)

$$
u_{j, j+1}=-h_{\mathrm{x}} \varphi_{j+1}-\frac{1}{2 x} h_{\mathrm{x}}^{2} \varphi_{j+1}-\frac{1}{2} h_{\mathrm{x}}^{2} \varphi_{j+1}^{2},
$$

or

$$
\varphi_{j+1}=-\frac{1}{h_{\mathrm{x}}}\left(1+\frac{1}{\Phi_{j+1}}\right)+\frac{1}{h_{\mathrm{x}}}\left[\left(1+\frac{1}{\Phi_{j+1}}\right)^{2}-2 u_{j, j+1}\right]^{\frac{1}{2}} .
$$

In much the same way, we derive

$$
u_{j+1, j}=h_{\mathrm{x}} \varphi_{j}-\frac{h_{\mathrm{x}}^{2}}{2 \Phi_{j+1}} \varphi_{j}-\frac{h_{\mathrm{x}}^{2}}{2} \varphi_{j}^{2},
$$

and Equation (12) implies

$$
u_{j, j}=0
$$

for $j>1$. At time $t=0$ when $x=s=a^{\prime}$, a combination of Equations (10) and (11) yields

$$
\begin{aligned}
\varphi_{1}=\left.\frac{\partial u}{\partial x}\right|_{t=0} & =\lim _{h_{\mathrm{x}} \rightarrow 0} \frac{u\left(x+h_{\mathrm{x}}, 0\right)-u(\mathrm{x}, 0)}{h_{\mathrm{x}}}-\frac{u_{1,1}}{h_{\mathrm{x}}} \\
& \cong \frac{u_{2,1}-u_{1,1}}{h_{\mathrm{x}}}=-\frac{u_{1,1}}{h_{\mathrm{x}}} .
\end{aligned}
$$

Furthermore, as is evident from Table I, and from Equation (A3) with $j=1$ we have

$$
\begin{gathered}
u_{1,1}=\frac{T_{0}}{u_{0}}, \\
u_{1,2}=\frac{\frac{h_{\mathrm{x}}}{h_{\mathrm{S}}} \varphi_{1} u_{1,1}+u_{2,2}}{\frac{h_{\mathrm{x}}}{h_{\mathrm{s}}} \varphi_{1}+1}=\frac{\frac{h_{\mathrm{x}}}{h_{\mathrm{s}}} \varphi_{1} u_{1,1}}{\frac{h_{\mathrm{x}}}{h_{\mathrm{S}}} \varphi_{1}+1} .
\end{gathered}
$$

With Equation (A2), Equation (A4) for $j=1$ yields $\varphi_{2}$. By integrating Equation (8) with Simpson's rule, we finally have

$$
\begin{gathered}
t_{1}=0, \\
t_{2}=\frac{h_{\mathrm{x}}}{3}\left(\frac{1}{\varphi_{2}}+\frac{4}{\varphi_{3 / 2}}+\frac{1}{\varphi_{1}}\right), \\
t_{j+1}=t_{j-1}+\frac{h_{\mathrm{x}}}{3}\left(\frac{1}{\varphi_{j+1}}+\frac{4}{\varphi_{j}}+\frac{1}{\varphi_{j-1}}\right)
\end{gathered}
$$

where $\varphi_{3 / 2}$ is given by linear interpolation between $\varphi_{1}$ and $\varphi_{2}$.

We now have all the necessary ingredients for a numerical solution of the stated initial boundary-value problem in cylindrical coordinates when the initial heat content in the metal specimen is given. We first compute $u_{1,1}$ with Equation (A7a), then obtain $\varphi_{1}$ from Equation (A 6 ), $u_{1,2}$ from Equation (A7b), and $\varphi_{2}$ from Equation (A4). With these quantities being determined, $\Gamma_{1}$ follows from Equation (A2), and $u_{i, 2}(i=1,2, \ldots)$ can be computed from Equations (A1), (A3), and (A5). The ensuing steps then only involve Equations (A1), (A2), (A3), and (A5) with the iteration suggested on $\Gamma$ and Equation (A8) to update the time. A solution for other symmetries is easily derived in the same way. In the case of the problem at fixed temperature, only the boundary condition (10) is changed, and Equation (A3) is replaced by 


$$
u_{1, j}=\frac{T_{0}}{u_{0}} \text {. }
$$

\section{APPENDIX B}

We construct here a numerical solution scheme for Equations (13)-(15). To solve Equation (13) numerically, we adopt the finite-difference scheme of Garofalo (unpublished, p. 17) and set

$$
\alpha^{-}=k\left(\frac{u_{i, j}+u_{i-1, j}}{2}\right)
$$

and

$$
\alpha^{+}=k\left(\frac{u_{i+1, j}+u_{i, j}}{2}\right) .
$$

Re-arranging and using Equation (14), Equations (13) and (15) imply the following tridiagonal system

$$
\begin{aligned}
& \alpha^{-} u_{i-1, j+1}+\left(\mathrm{r}_{j}+\alpha^{+}+\alpha^{-}+\frac{\alpha^{+}}{x / h_{\mathrm{x}}}\right) u_{\mathrm{i}, j+1}- \\
& -\left(\alpha^{+}+\frac{\alpha^{+}}{x / h_{\mathrm{x}}}\right) u_{i+1, j+1}= \\
& =\left(\alpha^{+}+\frac{\alpha^{+}}{x / h_{\mathrm{x}}}\right) u_{i+1, j}-\left(\alpha^{+}+\alpha^{-}+\frac{\alpha^{+}}{x / h_{\mathrm{x}}}\right) u_{i, j}- \\
& -\alpha^{-} u_{i-1, j}+\left(\alpha^{+}+\frac{\alpha^{+}}{x / h_{\mathrm{x}}}\right) u_{i+1, j-1}- \\
& -\left(\alpha^{+}+\alpha^{-}+\frac{\alpha^{+}}{x / h_{\mathrm{x}}}-\Gamma_{j}\right) u_{i, j-1}-\alpha^{-} u_{i-1, j-1}, \\
& u_{1, j}=\frac{T_{0}}{u_{0}} .
\end{aligned}
$$

The boundary conditions at $x=s$ are obtained by writing Equation (13) in the form

$$
\rho c \varphi \frac{\partial u}{\partial s}=\frac{k}{x} \frac{\partial u}{\partial x}+\frac{\partial k}{\partial u}\left(\frac{\partial u}{\partial x}\right)^{2}+k \frac{\partial^{2} u}{\partial x^{2}}
$$

and by employing the same reasoning as in section 2.2, explicitly

$$
\frac{\partial u}{\partial x}=\varphi,
$$

$$
\begin{aligned}
\frac{\partial u}{\partial s} & =-\varphi, \\
\frac{\partial^{2} u}{\partial x^{2}} & =-\frac{1}{k}\left(\rho c+\frac{\partial k}{\partial u}\right) \varphi^{2}-\frac{1}{x} \varphi .
\end{aligned}
$$

From these relations we deduce in much the same way as before

$$
\begin{aligned}
\varphi_{j+1}= & \frac{1}{h_{\mathrm{x}}} \frac{\left(1+h_{\mathrm{x}} / 2 x\right)}{\left(1+\frac{\partial k}{\partial u}\right)}+ \\
& +\frac{1}{h_{\mathrm{x}}} \frac{\left(\left(1+h_{\mathrm{x}} / 2 x\right)^{2}-2 u_{j, j+1}\left(\rho c+\frac{\partial k}{\partial u}\right) / k\right)^{\frac{1}{2}}}{\left(1+\frac{\partial k}{\partial u}\right)},
\end{aligned}
$$

$$
\begin{aligned}
& u_{j+1, j}=h_{\mathrm{x}} \varphi_{j}-\frac{1}{2} h_{\mathrm{x}}^{2}\left(\frac{\varphi_{j}}{x}+\frac{\rho c \varphi_{j}^{2}}{k}+\left.\frac{\varphi_{j}^{2}}{k} \frac{\partial k}{\partial u}\right|_{j}\right), \\
& u_{j, j-1}=h_{\mathrm{x}} \varphi_{j-1}-h_{\mathrm{x}}^{2}\left(\frac{\varphi_{j-1}}{x}+\frac{\rho c \varphi_{j+1}^{2}}{k}+\left.\frac{\varphi_{j-1}^{2}}{k} \frac{\partial k}{\partial u}\right|_{j-1}\right),
\end{aligned}
$$

$$
\begin{aligned}
& u_{j+1, j+1}= \\
& \quad=2 h_{\mathrm{x}} \varphi_{j-1}-2 h_{\mathrm{x}}^{2}\left(\frac{\varphi_{j-1}}{x}+\frac{\rho c \varphi_{j-1}^{2}}{k}+\left.\frac{\varphi_{j-1}^{2}}{k} \frac{\partial k}{\partial u}\right|_{j-1}\right) .
\end{aligned}
$$

When the equations for the insulating layer are solved (section 3.2), the new tridiagonal system is composed of Equations (B1) and

$$
\left(\frac{k\left(u_{1, j}\right)}{h_{\mathrm{x}}}-1\right) u_{1, j+1}-\frac{k\left(u_{1, j}\right)}{h_{\mathrm{x}}} u_{2, j+1}=-\frac{T_{0}}{u_{0}} .
$$

Equations (B3)-(B6) and (B7) also apply here, but the initial conditions are

$$
\begin{aligned}
& u_{1,1}=0, \\
& u_{1,2}=\left(\frac{T_{0}}{u_{0}}\right) /\left(1+k_{\mathrm{i}} / h_{\mathrm{x}}\right),
\end{aligned}
$$

from Equation (16), and

$$
\varphi_{1}=-\frac{T_{0}}{u_{0}},
$$

from Equations (14) and (16).

The system can then be solved with the same algorithm as before. 